\title{
Fast relapse and high drop out rate of 48 weeks daily interferon monotherapy in HIV-infected patients with chronic hepatitis $\mathbf{C}$ Raffaele Bruno*1, Paolo Sacchi ${ }^{1}$, Massimo Puoti ${ }^{2}$, Valentina Ciappina1, Cristina Zocchetti ${ }^{1}$, Enrico Brunetti ${ }^{1}$, Elena Maffezzini ${ }^{1}$, Anna Capelli1, Savino FA Patruno ${ }^{1}$, Antonello Malfitano ${ }^{1}$ and Gaetano Filice ${ }^{1}$
}

Address: ${ }^{1}$ Division of Infectious and Tropical Disease - IRCCS "San Matteo" Hospital, Pavia - University of Pavia, Pavia, Italy and ${ }^{2}$ Infectious and Tropical Disease Department - "Spedali Civili" Brescia Hospital, Brescia - University of Brescia, Brescia, Italy

E-mail: Raffaele Bruno* - r.bruno@smatteo.pv.it; Paolo Sacchi - paolo.sacchi1@tin.it; Massimo Puoti - m.puoti@iol.it; Valentina Ciappina - valentinaciappina@ hotmail.com; Cristina Zocchetti - amb.mit@smatteo.pv.it; Enrico Brunetti - selim@unipv.it; Elena Maffezzini - amb.mit@smatteo.pv.it; Anna Capelli - amb.mit@smatteo.pv.it; Savino FA Patruno - sfa.patruno@smatteo.pv.it; Antonello Malfitano - amalfitano@smatteo.pv.it; Gaetano Filice - g.filice@smatteo.pv.it

${ }^{*}$ Corresponding author

Published: 28 August 2002

BMC Infectious Diseases 2002, 2:17
Received: 26 April 2002

Accepted: 28 August 2002

This article is available from: http://www.biomedcentral.com/I47I-2334/2/17

(C) 2002 Bruno et al; licensee BioMed Central Ltd. This article is published in Open Access: verbatim copying and redistribution of this article are permitted in all media for any purpose, provided this notice is preserved along with the article's original URL.

Keywords: HIV, HCV, Coinfection, Interferon, Ribavirin

\begin{abstract}
Background: The standard of care for HCV Hepatitis is the combination of interferon (IFN) plus Ribavirin. In HIV patients the use of this combination therapy may induce drug interactions, and reduces the adherence to HAART.

The aim of this study is to evaluate safety and efficacy of a 48 weeks daily dose IFN schedule.

Methods: We evaluated 50 coinfected patients; alpha IFN 2a was administered at a dose of $3 \mathrm{MU}$ daily. The baseline values were the following : CD4+ 5 I 5 cells/mmc (mean); HIV-RNA $<50$ copies/ $\mathrm{ml}$ in all patients; HCV-RNA 28, $3 \times 106$ copies $/ \mathrm{ml}$.

Results: At 48 weeks, 10 patients (20\%) achieved a biochemical and virological response according to an intention to treat analysis.

Twenty four patients (48\%) underwent a drop-out mainly by side effects related to overlapping toxicity of interferon and antiretroviral therapy. All the patients, who responded to the treatment, showed a fast relapse one month after the end of treatment.

Conclusion: Although our results demonstrated a very poor outcome and a bad tolerance to interferon monotherapy, this approach should not be dropped out, mainly in patients at high risk for side effects and in those with cirrhosis who do not tolerate or are at increased risk for the use of ribavirin.
\end{abstract}

\section{Background}

HIV - hepatitis C virus (HCV) coinfection is common and affects more than one third of all HIV infected persons worldwide. Prevalence among risk categories varies ac- 
cording to shared risk factors for transmission, mainly Intravenous Drug Use (IDU) and haemophiliacs [1]. Chronic HCV infection seems to accelerate the course of HIV disease, resulting in a worsened clinical and immunological progression[2]. At the same time, several studies suggest that HIV disease modifies the natural history of HCV infection, leading to a faster course of progression from active hepatitis to cirrhosis, to end stage liver disease and death [3-6]. HCV infection mimics opportunistic diseases because its natural history is significantly accelerated in HIV patients [7].

Since 1996 Higly Active Antiretroviral therapy (HAART) has slowed the progression of HIV disease and decreased the rate of HIV associated mortality[8].

Because the prognosis of HIV disease has been modified by HAART, the need to treat HCV coinfection became a significant issue. However, few studies have described the use of Interferon- $\alpha$ for treatment of chronic HCV infection in HIV infected patients. The reports of Boyer [9] and Marriot [10] showed a poor sustained response. In the largest up to now published study, Soriano [11] described that the $20 \%$ of patients achieved a sustained virological response; in this study Interferon- $\alpha$ appeared to be well tolerated. These results have been obtained with a thrice weekly (TIW) Interferon schedule. Strategies to improve the response to therapy are needed to modify the progression of HCV related liver diseases. Combination therapy with Interferon alpha $2 \mathrm{~b}$ and ribavirin (RBV) is effective in immunocompetent patients, at least in $60 \%$ of those achieving an end of treatment virological response (ETR) and in $45 \%$ showing a sustained virological response (SVR) 6 months after stopping IFN and $\operatorname{RBV}(12,13)$. In mono infected patients with severe chronic hepatitis the percentage of SVR is about 35\% [14]. The need for a more aggressive therapy in HIV-HCV patients is justified by the worsened clinical course. A significant problem in the treatment of coinfection arises from the complexity of both HAART and standard of care regimen for HCV hepatitis. Indeed, the use of combination therapy with IFN plus ribavirin may induce drug interactions, (in vitro studies have demonstrated that ribavirin could affect intracellular phosphorylation of deoxynucleoside, mainly AZT, [15] leading to a reduced therapeutic effect), increases the pill burden, reducing the adherence to HAART, and the risk of mithocondrial toxicity $(16,17)$. In our previous study performed in immunocompetent patients we demonstrated that daily IFN administration is more effective than TIW[18].

On this basis we have attempted to treat coinfected patients with a daily interferon schedule, in order to improve the percentage of clinical response.
Table I: Baseline demographic characteristics of the patients

\begin{tabular}{ll}
\hline Number of patients & 50 \\
Males & 42 \\
females & 8 \\
Age (mean) & 37 \\
Past history of alchol abuse (\%) & $28(56)$ \\
Estimated duration of HCV & $29,2 \pm 13, \mathrm{I}$ \\
infection(months) [mean \pm SD] & \\
Antiretroviral Therapy (\%) & $45(91)$ \\
HCV genotypes & \\
Ib & 16 \\
$2-3$ & 25 \\
4 & 9 \\
Mean CD4 cells/mmc count (range) & $515(179-1135)$ \\
HIV-RNA & $<50 \mathrm{copie} / \mathrm{ml}$ in all patients \\
HCV-RNA (mean) & $28,3 \times 10^{6} \mathrm{copies} / \mathrm{ml}$ \\
Knodell score & $7 \pm 3$ \\
Cirrhosis (\%) & $7(14)$ \\
\end{tabular}

The aim of this pilot study is to evaluate the efficacy and safety of an aggressive daily schedule of $3 \mathrm{MU} \alpha$ Interferon in the treatment of HCV related chronic hepatitis in patients infected with HIV.

\section{Methods}

Fifty caucasian HIV-seropositive patients co-infected with HCV were enrolled in a study on the tolerance and efficacy of a daily administration of interferon- $\alpha$ for the treatment of active chronic hepatitis C. Patients were eligible for the study if they presented with detectable HCV RNA in serum, elevated serum alanine amino transferase (ALT) and a histological fibrosis score on liver biopsy of $\geq 1$. Histological necrotic inflammatory activity and fibrosis, were assessed quantitatively using the modified Knodell histological activity index [19] on a liver biopsy performed within 6 months prior entry into the study. Exclusion criteria were the presence of circulating Hbs antigen, a self reported daily intake of alcohol $>40$ gr, haemolytic disease, decompensated cirrhosis, auto-immune hepatitis, pregnancy and a diagnosis of opportunistic infections occurred in the 6 months before entry into the study. All patients gave informed consent prior to the inclusion in the study. The baseline demographic characteristics of the patient population are described in Table 1. Seventy seven percent of the patients had a past history of injecting drug use, with a mean duration of drug use of $18.2 \pm 3.3$ years. Forty five of the patients (91\%) had been receiving antiretroviral drugs for $3.0 \pm 1.5$ years at the time of inclusion into the study. Four of the antiretroviral-treated patients $(9 \%)$ were receiving a combination of two nucleoside reverse transcriptase inhibitors (NRTI). The other patients were taking a triple drug combination therapy including two NRTI and a protease inhibitor, indinavir $(\mathrm{n}=36)$ and 
saquinavir $(\mathrm{n}=5)$. All patients exhibited a positive PCR for HCV RNA in serum. Sixteen $(32 \%)$ carried virus of genotype la and lb; genotypes 2-3a were found in twenty five $(50 \%)$ whereas genotype 4 was found in nine patients $(18 \%)$. All patients $(100 \%)$ were naive to IFN. Upon inclusion into the study, all patients were given $3 \mathrm{MU}$ of $\alpha$ IFN 2a daily for 48 weeks. Interferon dose was reduced, when necessary to $1,5 \mathrm{MU}$ daily.

\section{Surrogate markers for hepatitis $C$ and HIV disease}

Serum levels of alanine amino transferase (ALT normal $\leq$ $39 \mathrm{IU} / \mathrm{l}$ ), aspartate aminotransferase (AST; normal, $\leq 34$ IU/l), gamma glutamyl transferase (GGT normal, $\leq 53 \mathrm{IU} /$ l) and bilirubin (normal $\leq 1.10 \mathrm{mg} / \mathrm{l}$ ) were measured using standard methods. Serum HCV RNA was measured by a qualitative PCR assay (COBAS Amplicor HCV, version 2.0, limit of detection of 100 copies/milliliter), and quantitative HCV RNA was evaluated by a branched DNA signal amplification assay test (Quantiplex HCV RNA 2.0, Chiron, Emeryville, California, USA; range of detection, $200.000-120.000 .000 \mathrm{geq} / \mathrm{ml}$ ). The HCV genotype was determined by hybridization of biotin-labelled PCR products to oligonucleotide probes bound to nitrocellulose membrane strips (Inno-Lipa; Innogenetics, Brussels, Belgium). Absolute numbers of $\mathrm{CD} 4$ cells in whole blood were determined by flow cytometry. Serum levels of HIVRNA were determined by the branched DNA test (Quantiplex HIV RNA 2.0; Chiron; threshold: 50 copies $/ \mathrm{ml}$ ). Both HIV-RNA levels and CD4 cells count were measured at baseline and after four, eight, twelve weeks and, subsequently every three months. Hepatic inflammation and fibrosis were graded using the modified Knodell histological activity index. The inflammation score was determined by combining the first free index scores: portal, periportal and lobular inflammation. The inflammation score has a range from 0 to 18 ; the higher score indicates more severe histological abnormalities. The degrees of fibrosis were: 0 , no fibrosis; 1 , portal fibrosis; 3 , bridging fibrosis and 4 cirrhosis.

\section{End Points}

The primary efficacy end point was a sustained virological response (SVR) defined as a lack of detectable HCV-RNA in the serum at 24 weeks after the treatment was completed. A secondary end point was the presence of a end of treatment response (ETR), defined as the absence of serum HCV-RNA at the end of 48 weeks of therapy. Adverse events, both clinical and laboratory, were checked at every scheduled visit.

\section{Statistical analysis}

Analysis of results was carried out using an as treated (where only patients carrying on treatment were included) and an intent to treat analysis, where missed data were considered as failure. Quantitative variables to compare baseline and follow-up values from individual patients were compared by using the $t$ test for paired samples, after log transformation, where appropriate. Results are expressed as mean \pm SD.

\section{Results}

We did not observe a reduction neither in absolute count nor in percent of CD4 cells. Only transient blips of HIV viremia were recorded with values less than 1000 copies/ $\mathrm{ml}$.

The mean ALT value in the study population were $169 \pm$ 129 at baseline. The necrotic-inflammatory and fibrosis scores. were $7 \pm 3$ and $2 \pm 1$, respectively. Seven out of the 50 patients showed histological evidence of cirrhosis (14\%). At baseline HCV viremia was $28.3 \pm 36.6 \times 10^{6}$ genome equivalents (geq) $/ \mathrm{ml}$. Only twenty six individuals completed 48 weeks of treatment without any serious or potentially life-threatening adverse events; there were no drug-related deaths. The mean duration of therapy was 23 \pm 19 weeks.

After 24 weeks of therapy, the mean levels of ALT decreased to $131 \pm 61 \mathrm{UI} / \mathrm{l}(p=N S)$. At this time HCV viremia was $9.2 \pm 12.4 \times 10^{6}$ genome equivalents (geq)/ $\mathrm{ml}(\mathrm{p}=0.66)$. The as treated analysis showed a percentage of responders of $58 \%(29 / 50)$. The intention to treat analysis showed a percentage of responders of $44 \%(22 / 50)$.

After 48 weeks of therapy, the mean levels of ALT decreased to $95 \pm 61 \mathrm{UI} / \mathrm{l}(p=N S)$. At this time HCV viremia was $1.4 \pm 1.6 \times 10^{6}$ genome equivalents $(\mathrm{geq}) / \mathrm{ml}(\mathrm{p}=$ $0.07)$. The percentage of responders decreased to $38 \%$ $(19 / 50)$ according to an as treated analysis, and to $20 \%$ (10/50) according to an intention to treat analysis. Among end of treatment virological responders 6 patients were genotype $3 \mathrm{~A}$, while 4 were genotype 1 . One of them was cirrhotic.

All out of the responders showed a rapid relapse within one months after completing the treatment : Alt mean value was $79 \pm 75 \mathrm{UI} / \mathrm{ml}(p=N S)$ and mean HCV-RNA value: $39.07 \pm 20,22 \times 10^{6}$ genome equivalents $(\mathrm{geq}) / \mathrm{ml}(p=$ NS). (table 2). Thus, the observed SVR rate was $0 \%$.

\section{Side effects}

Among patients who completed treatment, 4 had interferon dose reduction due to leukopenia; 2 out of the responder ones.

A total of 24 patients (48\%) discontinued the treatment because of therapy related side effects. The most frequent adverse event was depression, occurred in 8 patients $(33 \%)$. No patients were on antidepressant at the initiation of interferon. Nobody received a specific treatment 
Table 2: Laboratory findings at 24 weeks, end of treatment and one month after end of treatment.

\begin{tabular}{lcccccc}
\hline & Baseline & 24 weeks & P & $\begin{array}{c}\text { End of treatment } \\
\text { (ETR) I 2 months }\end{array}$ & P & $\begin{array}{c}\text { I month after end of } \\
\text { treatment }\end{array}$ \\
\hline ALT UI/ml (mean \pm SD) & $169 \pm 129$ & $131 \pm 61$ & NS & $95 \pm 61$ & NS & $79 \pm 75$ \\
$\begin{array}{l}\text { HCV-RNA } \times 10^{6} \text { geq/ml } \\
(\text { mean } \pm \text { SD) }\end{array}$ & $28.3 \pm 36.6$ & $9.2 \pm 12.4$ & 0.66 & $1.4 \pm 1.6$ & 0.07 & $39.07 \pm 20.22$ \\
\end{tabular}

for depression, which improved rapidly after discontinuation of interferon. Malaise, fatigue and fever, so strong to stop the therapy, were recorded in 8 patients $(33 \%)$. Leukopenia (absolute neutrophil count $<750 / \mathrm{mmc)}$ occurred in 6 patients (25\%) and did not improve after IFN dose reduction. Two patients were lost to follow up (8\%). Adherence to interferon therapy was difficult even in patients who did not experience side effects or did not necessitate a dose reduction. While patients were receiving IFN treatment, they have shown a reduced acceptance to antiretroviral drugs. (table 3) This means that the discomfort due to the concomitant treatment impaired the capacity to be adherent to antiretroviral drugs, leading to an increase of missed doses.

\section{Discussion}

HIV coinfection has been considered until a couple of years ago as an exclusion criteria in all the controlled trials on efficacy of interferon used as monotherapy and in combination with ribavirin. So, available data comes out from pilot studies or comparative studies versus HIV uninfected patients. Three hundred and eighty eight patients have been enrolled in the 12 studies published as full papers on interferon monotherapy. None of these studies was placebo controlled; both interferon alfa $2 \mathrm{a}$ and $2 \mathrm{~b}$ were used with doses ranging from 1 million units three times per week to 9 MIU daily. Arbitrarily cumulating these data, end of treatment response was obtained in $28 \%$ of treated patients and sustained response in $16 \%$. Ten per cent of patients dropped out[20]. In the largest published study, Soriano et al described how $20 \%$ of patients achieved a sustained virological response [11]. Summarizing the five studies, comparing sustained response rate in HIV seropositives and seronegatives we can identify a slightly lower rate of sustained response in HIV seropositives in all the trials; however this difference did not reach statistical significance in any of them [20].

Analyzing all these studies, ranked according to the year of publication, we can identify a sharp decrease in the rate of sustained response in the studies published in the last 3 years [21-23]. The change in response rate to interferon monotherapy could probably be related to a change of the
Table 3: Side effects leading to interruption

\begin{tabular}{lll}
\hline & $\begin{array}{l}\text { Number of patients } \\
\text { (total } \mathrm{n}=24)\end{array}$ & Percentage \\
\hline & & \\
Depression & 8 & $33 \%$ \\
Malaise, fatigue & 4 & $16 \%$ \\
Fever & 4 & $16 \%$ \\
Leukopenia & 6 & $25 \%$ \\
Lost to follow up & 2 & $8 \%$ \\
& & \\
\hline
\end{tabular}

kind of patients which have been included in the HAART era. Recently have become to be treated the most difficult patients with advanced fibrosis, unfavourable genotypes, high HCV viremia and lower CD4 counts.

Actually the standard of care for chronic hepatitis C in immunocompetent patients has become the combination therapy of interferon and ribavirin, but so far there are only few published data obtained in HIV-HCV coinfected patients with an impressive difference of sustained response rate among the studies [24][17][25].

However, the use of combination therapy with IFN plus ribavirin may induce drug interactions, (in vitro studies have demonstrated that ribavirin could affect intracellular phosphorylation of deoxynucleoside, mainly AZT,[15] and $\mathrm{d} 4 \mathrm{t}$, leading to a reduced therapeutic effect), increases the pill burden, compromising the adherence to HAART, and the risk of mithocondrial toxicity.

Indeed, recent reports suggest that addition of ribavirin to an NRTI based antiretroviral therapy may increase the risk of life-threatening lactic acidosis $[16,17]$.

The finding that NRTIs may interfere with mitochondrial DNA synthesis led to many studies evaluating these effects in vitro. These studies suggested a ranking of $\mathrm{ddC}>\mathrm{ddI}>\mathrm{d} 4 \mathrm{~T}>3 \mathrm{TC}>\mathrm{AZT}>\mathrm{ABC}$ for effects on mitochondrial polymerase gamma $[26,27]$. 
Because each NRTI has a different affinity for mitochondrial polymerase gamma, they could have a different capacity for inducing liver toxicity.

Hence, the choice of treatment of both hepatitis $\mathrm{C}$ and HIV must be oriented toward drugs with lower overlapping toxicity, mainly if ribavirin is used. This approach could be useful to increase the safety of therapy of coinfection and to avoid cases of fatal lactic acidosis. The use of interferon alone may reduce the incidence of such therapy related adverse events. In this setting we tried to use an aggressive daily interferon schedule (3 MU daily) which in immunocompetent patients gave us a good sustained response rate [18].

In the present study we report an ETR rate of $20 \%$ and a SVR rate of $0 \%$, with all patients relapsing one month after stopping the therapy. The ETR rate is comparable to that observed either in immunocompetent patients or in HIV-HCV coinfected patients treated with interferon alone three times weekly. This percentage of ETR observed in our study has been reduced by the high incidence of side effects due to the overlapping toxicity of both antiretroviral and interferon therapy. The SVR was 0\% and may probably be influenced, as observed in other studies with low SVR rate [21-23], by the lack of immune-clearance which regulates the late phase of viral decay. Indeed the more rapid is the viral decay, the higher is the probability of SVR [28].

Although a CD4 cell count threshold of less than 200 cells/mmc has been reported to reduce the likelihood of SVR, this has not been observed in our patients who did not respond, in spite of having more than 200 CD4 cells/ mmc. Most of the adverse events encountered in this study were similar to those reported in HIV seronegative immunocompetent patients, but they were emphasized by the concomitant administration of antiretrovirals which made the patients intolerant to both treatment, resulting in a very high drop out rate.

So far the optimal treatment schedule for HCV has not yet been established in co-infected patients. Several studies are ongoing to determine if the outcome could be improved by the use of pegylated interferon plus ribavirin, with promising preliminary results[29]. Data on pegylated interferon alone in HIV patients are actually lacking. Although our results have demonstrated a very poor outcome with interferon monotherapy, this approach should not be completely dropped out, especially using PEG Interferon, mainly in patients at high risk for side effects and in those with cirrhosis who do not tolerate or are at increased risk for the use of ribavirin. This strategy could be useful to increase the safety of therapy of coinfection and to avoid cases of severe liver toxicity and lactic acidosis.
However, depending upon the baseline CD4 cell count level, in patients with more than $350 \mathrm{CD} 4+/ \mathrm{mmc}$, hepatitis treatment should precede antiretroviral therapy to reduce the overall risk of toxicity.

\section{References}

I. Staples CT Jr, Rimland D, Dudas D: Hepatitis C in the HIV (human immunodeficiency virus) Atlanta V.A. (Veterans Affairs Medical Center) Cohort Study (HAVACS): the effect of coinfection on survival. Clin Infect Dis 1999, 29(1):150-4

2. Piroth L, Duong M, Quantin C, et al: Does hepatitis C virus co-infection accelerate clinical and immunological evolution of HIV-infected patients? AIDS 1998, I 2(4):38I-8

3. Eyster ME, Diamondstone LS, Lien JM, Ehmann WC, Quan S, Goedert JJ: Natural history of hepatitis $C$ virus infection in multitransfused hemophiliacs: effect of coinfection with human immunodeficiency virus. The Multicenter Hemophilia Cohort Study. J Acquir Immune Defic Syndr 1993, 6(6):602-10

4. Martin P, Di BA, Kassianides C, Lisker-Melman M, Hoofnagle J: Rapidly progressive non-A, non-B hepatitis in patients with human immunodeficiency virus infection. Gastroenterology 1989, 97(6): $|559-6|$

5. Benhamou $Y, D i M V$, Bochet $M$, et al: Factors affecting liver fibrosis in human immunodeficiency virus-and hepatitis $C$ viruscoinfected patients: impact of protease inhibitor therapy. Hepatology 200I, 34(2):283-7

6. Rockstroh J, Woitas R, Spengler U: Human immunodeficiency virus and hepatitis C virus coinfection. Eur J Med Res 1998, 3(6):269-77

7. Sulkowski MS, Mast EE, Seeff LB, Thomas DL: Hepatitis C virus infection as an opportunistic disease in persons infected with human immunodeficiency virus. Clin Infect Dis 2000, 30(Suppl I):S77-84

8. Palella F, Delaney K, Moorman A, et al: Declining morbidity and mortality among patients with advanced human immunodeficiency virus infection. HIV Outpatient Study Investigators. N Engl J Med 1998, 338(13):853-60

9. Boyer N, Marcellin P, Degott C, et al: Recombinant interferon-alpha for chronic hepatitis $C$ in patients positive for antibody to human immunodeficiency virus. Comite des Anti-Viraux. $J$ Infect Dis 1992, 165(4):723-6

10. Marriott E, Navas $S$, del RJ, et al: Treatment with recombinant alpha-interferon of chronic hepatitis $C$ in anti-HIV-positive patients. J Med Virol 1993, 40(2): 107-II

II. Soriano V, Garcia-Samaniego J, Bravo R, et al: Interferon alpha for the treatment of chronic hepatitis $C$ in patients infected with human immunodeficiency virus. Hepatitis-HIV Spanish Study Group. Clin Infect Dis 1996, 23(3):585-91

12. McHutchison J, Gordon S, Schiff E, et al: Interferon alfa-2b alone or in combination with ribavirin as initial treatment for chronic hepatitis C. Hepatitis Interventional Therapy Group. N Engl J Med 1998, 339(2I): 1485-92

13. Poynard T, Marcellin P, Lee S, et al: Randomised trial of interferon alpha2b plus ribavirin for $\mathbf{4 8}$ weeks or for $\mathbf{2 4}$ weeks versus interferon alpha2b plus placebo for $\mathbf{4 8}$ weeks for treatment of chronic infection with hepatitis C virus. International Hepatitis Interventional Therapy Group (IHIT). Lancet 1998, 352(9 |38): 1426-32

14. Poynard T, McHutchison J, Davis G, et al: Impact of interferon alfa-2b and ribavirin on progression of liver fibrosis in patients with chronic hepatitis C. Hepatology 2000, 32(5): | |3|-7

15. Vogt M, Hartshorn K, Furman P, et al: Ribavirin antagonizes the effect of azidothymidine on HIV replication. Science 1987, 235(4794): $1376-9$

16. Lafeuillade A, Hittinger G, Chadapaud S: Increased mitochondrial toxicity with ribavirin in HIV/HCV coinfection. Lancet 200I, 357(9252):280-I

17. Sauleda S, Juarez A, Esteban J, et al: Interferon and ribavirin combination therapy for chronic hepatitis $C$ in human immunodeficiency virus-infected patients with congenital coagulation disorders [In Process Citation]. Hepatology 200I, 34(5): $1035-40$

18. Bruno MD R, Sacchi P, Maffezzini E, Zara F, Brunetti E, Filice C, Patruno SFA, Filice G: Daily Interferon regimen for chronic hepatitis 
C. A prospective randomized study. Clin Drug Invest 1999, 18: I |16

19. Knodell RGIK, Black WC: Formulation and application of a numerical scoring system for assessing histological activity in asymptomatic chronic active hepatitis. Hepatology I 981, I:431435

20. Bonacini $M$, Puoti $M$ : Hepatitis $\mathbf{C}$ in patients with human immunodeficiency virus infection: diagnosis, natural history, metaanalysis of sexual and vertical transmission, and therapeutic issues. Arch Intern Med 2000, 160(22):3365-73

21. Prestileo T, Mazzola G, Di LF, et al: Response-adjusted alpha-interferon therapy for chronic hepatitis C in HIV-infected patients. Int J Antimicrob Agents 2000, I6(3):373-8

22. Bruno R, Sacchi P, Filice $C$, Filice G: Aggressive daily interferon therapy in HIV-HCV coinfected patients. J Acquir Immune Defic Syndr 2000, 25(4):372-3

23. Hayashi K, Fukuda $Y$, Nakano I, et al: Poor response to interferon treatment for chronic hepatitis $C$ in human immunodeficiency virus-infected haemophiliacs. Haemophilia 2000, 6(6):677-81

24. Landau A, Batisse D, Piketty $C$, et al: Long-term efficacy of combination therapy with interferon-alpha2b and ribavirin for severe chronic hepatitis C in HIV-infected patients [In Process Citation]. AIDS 200I, I5(16):2।49-55

25. Nasti G, Di GG, Tavio M, et al: Chronic hepatitis C in HIV infection: feasibility and sustained efficacy of therapy with interferon alfa-2b and tribavirin. AIDS 200I, 15(14): 1783-7

26. Kakuda T: Pharmacology of nucleoside and nucleotide reverse transcriptase inhibitor-induced mitochondrial toxicity. Clin Ther 2000, 22(6):685-708

27. White A: Mitochondrial toxicity and HIV therapy. Sex Transm $\operatorname{lnf} 2001,77: 158-173$

28. Wiley T, Briedi L, Lam N, Layden T: Early HCV RNA values after interferon predict response. Dig Dis Sci 1998, 43(10):2169-72

29. Soriano VG-SJ, Perez-Olmeda M, Barreiro P, Nunez M, RodriguezRosado R: Pegylated Interferon plus ribavirin for the treatment of chronic hepatitis $\mathbf{C}$ in HIV infected patients. Ist IAS Conference on HIV pathogenesis and treatment. Buenos Aires July 8-I I. 2001

\section{Pre-publication history}

The pre-publication history for this paper can be accessed here:

http://www.biomedcentral.com/1471-2334/2/17/prepub http.//www.biomedcentral.com/1471-2334/2/17 